\title{
Dynamic phase transition for the Taylor problem in the wide-gap case
}

\section{Zhibo Hou* and Tian Ma}

${ }^{*}$ Correspondence: hzb_303@126.com

Department of Mathematics, Sichuan University, Chengdu, 610064 , China

\section{Springer}

\begin{abstract}
The main objective of this paper is to study the stability and the type of transition of the Taylor problem in the wide-gap case by using the averaging method, and we conclude that the stability of the Taylor problem in the wide-gap case is essentially the same with that in the case of the narrow-gap. The main technical tools are the spectral theory for linear and completely continuous fields, the dynamic bifurcation theory and the transition theory for incompressible flows, both developed by Ma and Wang (Bifurcation Theory and Applications, 2005; Stability and Bifurcation of Nonlinear Evolution Equations, 2007).
\end{abstract}

Keywords: Taylor problem; spectral theory; dynamic bifurcation; transition theory

\section{Introduction}

In 1921, Taylor [1] observed and studied the stability of laminar flow, which is known as the Couette flow. Taylor studied the case, where the gap between two cylinders is smaller in comparison with the mean radius, and both of them rotate in the same direction. $\mathrm{He}$ found that when the Taylor number $T$ is smaller than a critical value $T_{c}>0$, called the critical Taylor number, the Couette flow is stable, and when the Taylor number crosses $T_{c}$, the Couette flow breaks out into a cellular pattern which is radially symmetric.

Since Taylor's work, there have been many studies on this kind of problem. Such as Couette [2] and Mallock [3] did a lot of experiments. Cloes [4] published the most comprehensive experimental results associated with the Taylor vortex and the secondary instability. Chandrasekhar [5], Walowit et al. [6], Drazin and Reid [7] studied the linear theories. Velte [8], Kirchgässner [9], Kirchgässner and Sorger [10], Yudovich [11], Ma and Wang [12-14] studied the nonlinear theories. Especially, Ma and Wang established a new notion of bifurcation, called an attractor bifurcation, which was applied to the Taylor problem and obtained a series of fine results. This paper focuses on the Taylor problem in the wide-gap case. In this case, the radius of inner cylinder is small, while the radius of the outer cylinder is big. In addition to the same direction, the two cylinders could rot in the converse direction.

The main objective is to study the stability and the type of transition of the Taylor problem in the wide-gap case by using the averaging method and to compare with the Taylor problem in the narrow-gap case.

The main technical tools are the spectral theory for linear and completely continuous fields, the dynamic bifurcation theory and the transition theory for incompressible flows. These theories are directly applied to the Taylor problem in the wide-gap case.

@2013 Hou and Ma; licensee Springer. This is an Open Access article distributed under the terms of the Creative Commons Attribution License (http://creativecommons.org/licenses/by/2.0), which permits unrestricted use, distribution, and reproduction in any medium, provided the original work is properly cited. 
The main conclusion is that the stability of the Taylor problem in the wide-gap case is essentially the same with that in the case of a narrow-gap. The main theorems are presented in Section 4, through which we can give pictures depicting the Couette flow stability in the wide-gap case, and compare with the Taylor problem in the narrow-gap case. In the later research, we intend to simulate the Taylor problem in the wide-gap case by using computer.

This paper is organized as follows. Section 2 introduces the governing equations for the Taylor problem. Section 3 studies the Taylor problem in the wide-gap case by using the averaging method, and establishes its mathematical frame. All the main theorems and the proofs are presented in Section 4.

\section{Governing equations for the Taylor problem}

The governing equations for the Taylor problem are the Navier-Stocks equations in the cylindrical coordinates $(z, r, \theta)$, which have the form

$$
\left\{\begin{array}{l}
\frac{\partial u_{z}}{\partial t}+(u \cdot \nabla) u_{z}=-\frac{\partial}{\partial z}\left(\frac{p}{\rho}\right)+v \Delta u_{z}, \\
\frac{\partial u_{r}}{\partial t}+(u \cdot \nabla) u_{r}-\frac{u_{\theta}^{2}}{r}=-\frac{\partial}{\partial r}\left(\frac{p}{\rho}\right)+v\left(\Delta u_{r}-\frac{2}{r^{2}} \frac{\partial u_{\theta}}{\partial \theta}-\frac{u_{r}}{r^{2}}\right), \\
\frac{\partial u_{\theta}}{\partial t}+(u \cdot \nabla) u_{\theta}+\frac{u_{r} u_{\theta}}{r}=-\frac{\partial}{\partial \theta}\left(\frac{p}{\rho}\right)+v\left(\Delta u_{\theta}+\frac{2}{r^{2}} \frac{\partial u_{r}}{\partial \theta}-\frac{u_{\theta}}{r^{2}}\right), \\
\frac{\partial\left(r u_{r}\right)}{\partial r}+\frac{\partial u_{\theta}}{\partial \theta}+\frac{\partial\left(r u_{z}\right)}{\partial z}=0,
\end{array}\right.
$$

where $v$ is the kinematic viscosity, $\rho$ the density, $u=\left(u_{z}, u_{r}, u_{\theta}\right)$ the velocity field, $p$ the pressure function, and

$$
\begin{aligned}
& u \cdot \nabla=u_{z} \frac{\partial}{\partial z}+u_{r} \frac{\partial}{\partial r}+\frac{u_{\theta}}{r} \frac{\partial}{\partial \theta}, \\
& \Delta=\frac{\partial^{2}}{\partial z^{2}}+\frac{1}{r} \frac{\partial}{\partial r}\left(r \frac{\partial}{\partial r}\right)+\frac{1}{r^{2}} \frac{\partial^{2}}{\partial \theta^{2}} .
\end{aligned}
$$

The basic flow for (2.1) is the Couette flow, namely a steady state solution defined by

$$
\begin{aligned}
& u_{z}=u_{r}=0, \quad u_{\theta}=V(r), \quad p=\rho \int \frac{1}{r} V^{2}(r) d r, \\
& V(r)=a r+\frac{b}{r},
\end{aligned}
$$

where

$$
\begin{aligned}
& a=-\Omega_{1} \eta^{2} \frac{1-\mu / \eta^{2}}{1-\eta^{2}}, \quad b=\Omega_{1} \frac{r_{1}^{2}(1-\mu)}{1-\eta^{2}}, \\
& \mu=\frac{\Omega_{2}}{\Omega_{1}}, \quad \eta=\frac{r_{1}}{r_{2}} .
\end{aligned}
$$

In order to investigate the stability of the Couette flow defined by (2.1), we need to consider the perturbed state

$$
u_{z}, \quad u_{r}, \quad u_{\theta}+V(r), \quad p+\rho \int \frac{1}{r} V^{2}(r) d r
$$


Assume that the perturbations are axisymmetric and independent of $\theta$, from (2.1), we have:

$$
\left\{\begin{array}{l}
\frac{\partial u_{z}}{\partial t}+(\tilde{u} \cdot \nabla) u_{z}=v \Delta u_{z}-\frac{\partial}{\partial z}\left(\frac{p}{\rho}\right), \\
\frac{\partial u_{r}}{\partial t}+(\tilde{u} \cdot \nabla) u_{r}-\frac{u_{\theta}^{2}}{r}=v\left(\Delta u_{r}-\frac{u_{r}}{r^{2}}\right)-\frac{\partial}{\partial r}\left(\frac{p}{\rho}\right)+\frac{2 V}{r} u_{\theta}, \\
\frac{\partial u_{\theta}}{\partial t}+(\tilde{u} \cdot \nabla) u_{\theta}+\frac{u_{r} u_{\theta}}{r}=v\left(\Delta u_{\theta}-\frac{u_{\theta}}{r^{2}}\right)-\left(V^{\prime}+\frac{V}{r}\right) u_{r} \\
\frac{\partial\left(r u_{r}\right)}{\partial r}+\frac{\partial\left(r u_{z}\right)}{\partial z}=0
\end{array}\right.
$$

where

$$
\begin{aligned}
& \tilde{u} \cdot \nabla=u_{z} \frac{\partial}{\partial z}+u_{r} \frac{\partial}{\partial r} \\
& \Delta=\frac{\partial^{2}}{\partial z^{2}}+\frac{\partial^{2}}{\partial r^{2}}+\frac{1}{r} \frac{\partial}{\partial r} .
\end{aligned}
$$

The spatial domain for (2.3) is $M=\left(r_{1}, r_{2}\right) \times(0, L) \subset R^{2}$, where $L$ is the height of the field between the two cylinders. There are different physically sound boundary conditions.

In the radial direction, there are two kinds of boundary conditions:

Free boundary condition:

$$
\frac{\partial u_{z}}{\partial r}=0, \quad u_{r}=u_{\theta}=0, \quad \text { at } r=r_{1}, r_{2} .
$$

Rigid boundary condition:

$$
u_{z}=u_{r}=u_{\theta}=0, \quad \text { at } r=r_{1}, r_{2} .
$$

In the $z$ direction, there are four kinds of boundary conditions:

Free slip boundary condition:

$$
u_{z}=0, \quad \frac{\partial u_{r}}{\partial z}=\frac{\partial U_{\theta}}{\partial z}=0, \quad \text { at } z=0, L .
$$

Dirichlet boundary condition (or rigid condition):

$$
u_{z}=u_{r}=u_{\theta}=0, \quad \text { at } z=0, L .
$$

Free rigid boundary condition:

$$
\begin{aligned}
& u_{z}=0, \quad \frac{\partial u_{r}}{\partial z}=\frac{\partial u_{\theta}}{\partial z}=0, \quad \text { at } z=L, \\
& u_{z}=u_{r}=u_{\theta}=0, \quad \text { at } z=0 .
\end{aligned}
$$

Periodic condition:

$u=\left(u_{z}, u_{r}, u_{\theta}\right)$ is periodic in the $z$ direction. 
Using the transform:

$$
\left\{\begin{array}{l}
x=l x^{\prime}, \quad x=(z, r, \theta) \\
t=l^{2} t^{\prime} / v \\
u=v u^{\prime} / l, \quad u=\left(u_{z}, u_{r}, u_{\theta}\right) \\
p=\rho v^{2} p^{\prime} / l^{2}
\end{array}\right.
$$

where $l$ is a certain length unit in (2.3).

We get the dimensionless form:

$$
\left\{\begin{array}{l}
\frac{\partial u_{z}}{\partial t}=\Delta u_{z}-\frac{\partial p}{\partial z}-(\tilde{u} \cdot \nabla) u_{z}, \\
\frac{\partial u_{r}}{\partial t}=\left(\Delta u_{r}-\frac{u_{r}}{r^{2}}\right)-\frac{\partial p}{\partial r}+2 \Omega_{1} \frac{l^{2}}{v}\left(\frac{r_{1}^{2}}{r^{2}} \frac{1-\mu}{1-\eta^{2}}-\frac{\eta^{2}-\mu}{1-\eta^{2}} u_{\theta}\right)+\frac{u_{\theta}^{2}}{r}-(\tilde{u} \cdot \nabla) u_{r}, \\
\frac{\partial u_{\theta}}{\partial t}=\left(\Delta u_{\theta}-\frac{u_{\theta}}{r^{2}}\right)+2 \Omega_{1} \frac{l^{2}}{v} \frac{\eta^{2}-\mu}{1-\eta^{2}} u_{r}-\frac{u_{r} u_{\theta}}{r}-(\tilde{u} \cdot \nabla) u_{\theta}, \\
\frac{\partial\left(r u_{r}\right)}{\partial r}+\frac{\partial\left(r u_{z}\right)}{\partial z}=0 .
\end{array}\right.
$$

\section{Averaging for the Taylor problem in the wide-gap case}

In the case of a wide-gap, $r_{1}$ is small, while $r_{2}$ is big. The averaging method is that we let $l=r_{1}=1$, and $r_{0}=\left(r_{1}+r_{2}\right) / 2$ replace variable $r$ approximately. Since $r_{x}$ is big, $r_{1}$ and $v$ are small, we can ignore $r^{-n}(n \geq 1)$, which are associated with the inner friction of fluid. In this paper, moreover, our analysis will be conducted using free boundary conditions in $z$ and radial directions. Then from (2.4), we obtain the averaged governing equation for the case of the wide-gap:

$$
\begin{aligned}
& \left\{\begin{array}{l}
\frac{\partial u_{z}}{\partial t}=\Delta u_{z}-\frac{\partial p}{\partial z}-(\tilde{u} \cdot \nabla) u_{z}, \\
\frac{\partial u_{r}}{\partial t}=\Delta u_{r}-\frac{\partial p}{\partial r}+\lambda\left(\frac{1}{r_{0}^{2}}-\kappa\right) u_{\theta}+\frac{u_{\theta}^{2}}{r_{0}}-(\tilde{u} \cdot \nabla) u_{r}, \\
\frac{\partial u_{\theta}}{\partial t}=\Delta u_{\theta}+\lambda \kappa u_{r}-\frac{u_{r} u_{\theta}}{r_{0}}-(\tilde{u} \cdot \nabla) u_{\theta}, \\
\frac{\partial u_{r}}{\partial r}+\frac{\partial u_{z}}{\partial z}=0,
\end{array}\right. \\
& \begin{cases}\frac{\partial u_{z}}{\partial r}=0, & u_{r}=u_{\theta}=0, \quad \text { at } r=1, r_{2} \\
u_{z}=0, \quad \frac{\partial u_{r}}{\partial z}=\frac{\partial u_{\theta}}{\partial z}=0, \quad \text { at } z=0, L\end{cases}
\end{aligned}
$$

where $\lambda=\sqrt{T}, T$ is the Taylor number, $T$ and $\kappa$ are defined by

$$
\begin{aligned}
& T=\frac{4 \Omega_{1}^{2}\left(1-\mu^{2}\right)}{v^{2}\left(1-\eta^{2}\right)}, \quad \kappa=\frac{\eta^{2}-\mu}{1-\mu}, \\
& \tilde{u} \cdot \nabla=u_{z} \frac{\partial}{\partial z}+u_{r} \frac{\partial}{\partial r}, \\
& \Delta=\frac{\partial^{2}}{\partial z^{2}}+\frac{\partial^{2}}{\partial r^{2}},
\end{aligned}
$$

spatial domain for (3.1) is $M=\left(1, r_{2}\right) \times(0, L) \subset R^{2}$.

Let $\tilde{u}=\left(u_{z}, u_{r}\right)$,

$$
\begin{aligned}
& H=\left\{u=\left(\tilde{u}, u_{\theta}\right) \in L^{2}\left(M, R^{3}\right)|\operatorname{div} \tilde{u}=0, \tilde{u} \cdot n|_{\partial M}=0\right\}, \\
& H_{1}=\left\{u=\left(\tilde{u}, u_{\theta}\right) \in H^{2}\left(M, R^{3}\right) \cap H \mid u \text { satisfies (3.2) }\right\} .
\end{aligned}
$$


By the Hodge decomposition theorem, $L^{2}\left(M, R^{3}\right)$ can be decomposed as follows.

$$
\begin{aligned}
& L^{2}\left(M, R^{3}\right)=H \oplus G, \\
& H=\left\{u=\left(\tilde{u}, u_{\theta}\right) \in L^{2}\left(M, R^{3}\right)|\operatorname{div} \tilde{u}=0, \tilde{u} \cdot n|_{\partial M}=0\right\}, \\
& G=\left\{\nabla_{\varphi} \in L^{2}\left(M, R^{3}\right) \mid \varphi \in H^{1}(M)\right\}, \\
& G \perp H .
\end{aligned}
$$

Here, $H^{1}(M)$ and $H^{2}\left(M, R^{3}\right)$ are the usual Sobolev spaces.

Then we can define an orthogonal projection, called Leray projection

$$
P: L^{2}\left(M, R^{3}\right) \rightarrow H
$$

Let

$$
\begin{aligned}
& L_{\lambda}=-A+\lambda B: H_{1} \rightarrow H, \\
& G: H_{1} \rightarrow H
\end{aligned}
$$

be a mapping defined by

$$
\begin{aligned}
& A(u)=-P\left(\Delta u_{z}, \Delta u_{r}, \Delta u_{\theta}\right), \\
& B(u)=P\left(0,\left(\frac{1}{r_{0}^{2}}-\kappa\right) u_{\theta}, \kappa u_{r}\right), \\
& G(u)=-P\left((\tilde{u} \cdot \nabla) u_{z},(\tilde{u} \cdot \nabla) u_{r}-\frac{u_{\theta}^{2}}{r_{0}},(\tilde{u} \cdot \nabla) u_{\theta}+\frac{u_{\theta} u_{r}}{r_{0}}\right),
\end{aligned}
$$

where $u=\left(u_{z}, u_{r}, u_{\theta}\right) \in H_{1}$, and the mapping $P$ is the Leray projection.

Thus, (3.1) can be written in the abstract form:

$$
\frac{d u}{d t}=L_{\lambda} u+G u, \quad u \in H_{1} .
$$

So far, the stability of the Couette flows of equation (2.1) equals to the stability of $\left(u_{z}, u_{r}, u_{\theta}\right)=0$ of equation (3.4).

\section{Main results and proofs}

Let $X_{1}, X$ be two Banach spaces, $X_{1} \subset X$ be a dense and compact inclusion. Consider the following nonlinear evolution equations

$$
\left\{\begin{array}{l}
\frac{d u}{d t}=L u+G u, \\
u(0)=\varphi,
\end{array}\right.
$$

where $L: X_{1} \rightarrow X$ is bounded linear operator, $G: X_{1} \rightarrow X$ is $C^{r}(r \geq 1)$ mapping.

Definition 4.1 [14] Let $\Omega \subset X$ be a bounded open set, we say that (4.1) is Lyapunov stable in $\Omega$, if the solution of (4.1) $u(t, \varphi) \in \Omega, \forall t \geq 0$, for any initial point $\varphi \in \Omega$. 
Definition 4.2 [14] We say (4.1) is asymptotically Lyapunov stable in $\Omega$, or $u_{0}$ is an asymptotically stable equilibrium point of (4.1) in $\Omega$, if there exists a steady solution $u_{0} \in \Omega$, satisfying

$$
\lim _{t \rightarrow \infty}\left\|u(t, \varphi)-u_{0}\right\|_{X}=0 \quad \text { for any } \varphi \in \Omega
$$

Lemma 4.3 [14] Let $L: X_{1} \rightarrow x$ be a sectorial operator, $G: X_{\alpha} \rightarrow X$ be a $C^{r}(r \geq 1)$ mapping for certain $0 \leq \alpha<1, v$ be a steady solution of (4.1). If the spectral $\beta(\lambda)$ of $L+D G(v)$ satisfy $\operatorname{Re} \beta(\lambda)<-\varepsilon$, for certain $\varepsilon>0$, then $v$ is a locally asymptotically stable equilibrium point of (4.1), and it decays exponentially. Namely, there exist $M>0, \sigma>0, \delta>0$, for the solution of (4.1) $u(t, \varphi),\|u(t, \varphi)-v\|_{X} \leq M e^{-\sigma t}(\forall t \geq 0)$ hold true, as $\|\varphi-v\|<\delta$.

Let

$$
\delta=\frac{3 r_{2}^{2}-2 r_{2}-1}{r_{2}^{2}\left(r_{2}-1\right)\left(r_{2}+3\right)}>0, \quad \text { as } r_{2}>r_{1}=1 \text {, }
$$

and as above

$$
\mu=\frac{\Omega_{2}}{\Omega_{1}}, \quad \eta=\frac{r_{1}}{r_{2}} .
$$

Theorem 4.4 If $\mu \leq-\delta$, or $\eta^{2} \leq \mu$, then $\left(u_{z}, u_{r}, u_{\theta}\right)=0$ is a locally asymptotically stable equilibrium point of (3.4), and it decays exponentially.

Theorem 4.5 If $-\delta<\mu<\eta^{2}(\mu \neq 0)$, we have the following conclusions for (3.4):

(1) Equation (3.4) happens with a continuous transition at $(u, \lambda)=\left(0, \sqrt{T_{c}}\right)$, namely there is an attractor bifurcation, and it bifurcates exactly into two singular points $v_{i}$ $(i=1,2)$, which attract two open subsets of $U$ separately. $U$ is the neighborhood of $u=0$.

(2) The two singular points $v_{i}(i=1,2)$ can be written by:

$$
v_{1,2}(\lambda)= \pm\left|\beta_{n_{1} 1} / \sigma\right|^{\frac{1}{2}} \psi_{n_{1} 1}+o\left(\left|\beta_{n_{1} 1} / \sigma\right|^{\frac{1}{2}}\right)
$$

where $\sigma=\frac{-1}{4 \beta_{2 n_{1} 2}} a_{1}^{4} b_{1}^{2}, \beta_{n_{1} 1}$ is the first eigenvalue.

Remark 4.6 From the point of view of mathematics, Theorem 4.4 explains that there exists an open set $\Omega \subset H_{1}$, which guarantees that if the initial point $u_{0} \in \Omega$, then the solution of (3.4) satisfies $\left\|u\left(t, u_{0}\right)\right\|_{H} \rightarrow 0(t \rightarrow \infty)$.

Remark 4.7 From the point of view of physics, Theorem 4.4 explains that in the wide-gap case, the Couette flow of the Taylor problem is metastable. Namely, if the initial perturbation is in a certain range, the disturbed fluid will become the Couette flow in a short time. But if the initial perturbation is beyond that range above, the disturbed fluid will become another steady flow. The explanation is the same as that for Theorem 4.5.

Remark 4.8 Theorems 4.4-4.5 give the entire results of stability of the Taylor problem in the wide-gap case; see Figure 1. $\Sigma_{1}$ represents the unstable area, $\Sigma_{2}$ represents the stable 
Figure 1 Wide-gap Case.

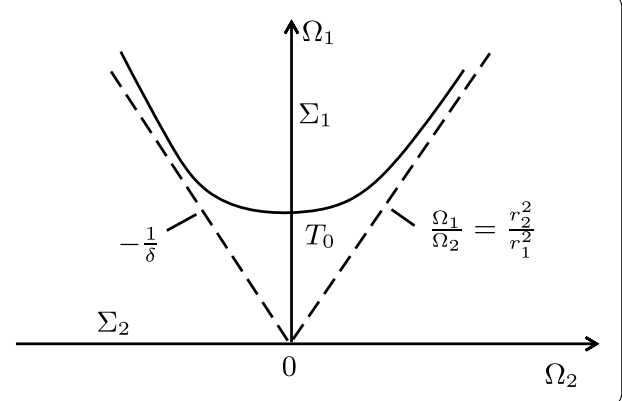

Figure 2 Narrow-gap Case.

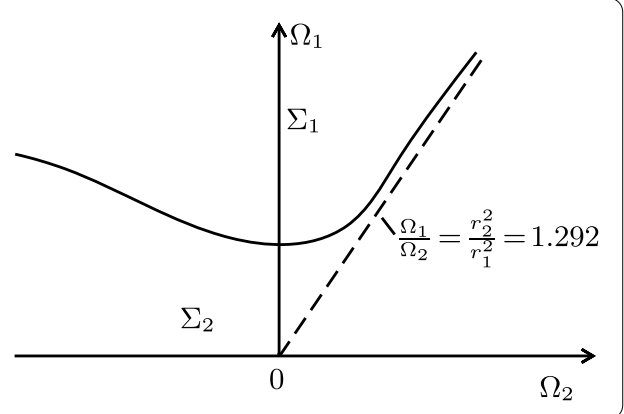

area, $T_{0} \neq 0$ and

$$
T_{0}=\min _{(n, l)} \frac{\alpha^{3} v^{2}\left(1-\eta^{2}\right)^{2}}{4 a^{2} \eta^{2}\left(\frac{1}{r_{0}^{2}}-\eta^{2}\right)}
$$

Remark 4.9 [15] In the narrow-gap case, let $r_{1}=3.55 \mathrm{~cm}$ and $r_{2}=4.035 \mathrm{~cm}$, the results can be seen in Figure 2. $\Sigma_{1}$ represents the unstable area, $\Sigma_{2}$ represents the stable area.

Remark 4.10 By Figure 1 and Figure 2, we can conclude that the results of the stability of the Taylor problem in the wide-gap case, obtained by using the averaging method, are essentially the same as those of the Taylor problem in the narrow-gap case.

Proof of Theorem 4.4 Obviously, $A$ and $B$ are linear operators. According to [16], $A$ is a homeomorphism, then it is a sectorial operator. According to the Sobolev compact embedding theorem [17] and the Leray projection, $P$ is bounded, $B$ is compact. So,

$$
L_{\lambda}=-A+\lambda B: H_{1} \rightarrow H
$$

is a sectorial operator, and also a linear completely continuous field.

Now,

$$
\begin{aligned}
\int_{\Omega}(u \cdot \nabla) u \cdot v+u^{2} \cdot v d x & \leq\|u\|_{C^{0}}\|\nabla u\|_{L^{2}}\|v\|_{L^{2}}+\|u\|_{C^{0}}\|u\|_{L^{2}}\|v\|_{L^{2}} \\
& =\|u\|_{C^{0}}\|u\|_{W^{1,2}}\|v\|_{L^{2}},
\end{aligned}
$$


we have

$$
\|G u\|_{H} \leq\|u\|_{C^{0}}\|u\|_{W^{1,2}} .
$$

According to [18], Theorem 7.2.2, the fraction spaces of $L_{\lambda}, H_{\alpha}=D\left(L_{\lambda}^{\alpha}\right)$, satisfy

$$
\|u\|_{C^{0}}+\|u\|_{W^{1,2}} \leq C\|u\|_{H_{\alpha}}
$$

where $\alpha>\frac{3}{4}$. Then

$$
G: H_{\alpha} \rightarrow H \quad\left(\frac{3}{4}<\alpha<1\right) \text { is a bounded analytic mapping. }
$$

Consider the eigenvalue problem:

$$
L_{\lambda} \psi=\beta(\lambda) \psi, \quad \psi=\left(u_{z}, u_{r}, u_{\theta}\right) \in H_{1} .
$$

By (3.3), the abstract form can be referred to the following eigenvalue equations in $H_{1}$ :

$$
\begin{aligned}
& \Delta u_{z}-\frac{\partial p}{\partial z}=\beta(\lambda) u_{z}, \\
& \Delta u_{r}-\frac{\partial p}{\partial r}+\lambda\left(\frac{1}{r_{0}^{2}}-\kappa\right) u_{\theta}=\beta(\lambda) u_{r}, \\
& \Delta u_{\theta}+\lambda \kappa u_{r}=\beta(\lambda) u_{\theta}, \\
& \frac{\partial u_{r}}{\partial r}+\frac{\partial u_{z}}{\partial z}=0, \\
& \frac{\partial u_{z}}{\partial r}=0, \quad u_{r}=u_{\theta}=0, \quad \text { at } r=1, r_{2}, \\
& u_{z}=0, \quad \frac{\partial u_{r}}{\partial z}=\frac{\partial u_{\theta}}{\partial z}=0, \quad \text { at } z=0, L .
\end{aligned}
$$

According to [5], we take the separation of variables as follows:

$$
\begin{aligned}
& u_{z}=\frac{d h(z)}{d t} \frac{d R(r)}{d r}, \\
& u_{r}=a^{2} h(z) R(r), \\
& u_{\theta}=h(z) \varphi(r) .
\end{aligned}
$$

We utilize (4.8) in (4.5) and (4.7) to obtain:

$$
\left\{\begin{array} { l } 
{ h ^ { \prime \prime } R ^ { \prime } + a ^ { 2 } h R ^ { \prime } = 0 , } \\
{ h ^ { \prime } ( 0 ) = h ^ { \prime } ( L ) = 0 }
\end{array} \quad \Rightarrow \left\{\begin{array}{l}
h=\cos a z, \\
a=\frac{n \pi}{L}, \quad n=1,2, \ldots
\end{array}\right.\right.
$$

We utilize (4.8), (4.9) in (4.4) to obtain:

$$
h^{\prime \prime} \varphi+h \varphi^{\prime \prime}+\lambda \kappa a^{2} h R=\beta(\lambda) h \varphi
$$


that equals to

$$
\left(\frac{d^{2}}{d r^{2}}-a^{2}\right) \varphi=-\lambda \kappa a^{2} R+\beta(\lambda) \varphi .
$$

We utilize (4.8), (4.9) in (4.2), then differentiate with respect to $r$,

$$
h^{\prime \prime \prime} R^{\prime \prime}+h^{\prime} R^{\prime \prime \prime \prime}-\frac{\partial^{2} p}{\partial r \partial z}=\beta(\lambda) h^{\prime} R^{\prime \prime},
$$

and utilize (4.8), (4.9) in (4.3), then differentiate with respect to $z$,

$$
a^{2} h^{\prime \prime \prime} R+a^{2} h^{\prime} R^{\prime \prime}+\lambda\left(\frac{1}{r_{0}^{2}}-\kappa\right) h^{\prime} \varphi-\frac{\partial^{2} p}{\partial z \partial r}=\beta(\lambda) a^{2} h^{\prime} R,
$$

the two equations above subtract to obtain:

$$
\left(\frac{d^{2}}{d r^{2}}-a^{2}\right)^{2} R=\lambda\left(\frac{1}{r_{0}^{2}}-\kappa\right) \varphi+\beta(\lambda)\left(\frac{d^{2}}{d r^{2}}-a^{2}\right) R
$$

Utilizing (4.8) in (4.6), we have

$$
\varphi(1)=\varphi\left(r_{2}\right)=R(1)=R\left(r_{2}\right)=R^{\prime \prime}(1)=R^{\prime \prime}\left(r_{2}\right)=0,
$$

then let

$$
R=\sin l \pi\left(\frac{r-1}{r_{2}-1}\right), \quad \varphi=C_{n l} \sin l \pi\left(\frac{r-1}{r_{2}-1}\right), \quad l=1,2, \ldots
$$

Combine (4.10), (4.11) to obtain:

$$
\left\{\begin{array}{l}
\alpha C_{n l}=a^{2} \lambda \kappa-C_{n l} \beta(\lambda), \\
\alpha^{2}=C_{n l}\left(\frac{1}{r_{0}^{2}}-\kappa\right) \lambda-\alpha \beta(\lambda), \quad \alpha=a^{2}+\left(\frac{l \pi}{r_{2}-1}\right)^{2},
\end{array}\right.
$$

therefore, we get

$$
\left\{\begin{array}{l}
C_{n l}=\frac{2 \alpha \lambda \kappa a^{2}}{ \pm \sqrt{\Delta}} \\
\beta_{n l}=-\alpha \pm \frac{\sqrt{\Delta}}{2 \alpha}
\end{array}\right.
$$

where

$$
\Delta=4 \alpha a^{2} \lambda^{2} \kappa\left(\frac{1}{r_{0}^{2}}-\kappa\right) .
$$

The corresponding eigenvectors of (4.2)-(4.7) are as follows:

$$
\psi_{n l}=\left\{\begin{array}{l}
\frac{n l \pi^{2}}{L\left(r_{2}-1\right)} \sin \frac{n \pi z}{L} \cos \frac{l \pi(r-1)}{\left(r r_{2}-1\right)}, \\
\left(\frac{n \pi}{L}\right)^{2} \cos \frac{n \pi z}{L} \sin \frac{l \pi(r-1)}{\left(r_{2}-1\right)}, \\
C_{n l} \cos \frac{n \pi z}{L} \sin \frac{l \pi(r-1)}{\left(r_{2}-1\right)} .
\end{array}\right.
$$


Now, as $(n, l) \neq(0,0)$,

$$
\begin{aligned}
& L_{\lambda} \psi_{n l}=\beta_{n l}(\lambda) \psi_{n l}, \\
& E_{1}=\left\{\psi_{n l} \mid \psi_{n l} \text { satisfies }(4.14),(n, l) \in N \times N\right\}, \\
& B_{1}=\left\{\beta_{n l}(\lambda) \mid \beta_{n l}(\lambda) \text { satisfies }(4.13),(n, l) \in N \times N\right\},
\end{aligned}
$$

as $n=0$, from (4.2)-(4.8),

$$
L_{\lambda} \psi_{0 l}=\beta_{0 l}(\lambda) \psi_{0 l},
$$

where

$$
\begin{aligned}
& \psi_{0 l} \in E_{2}=\left\{\psi_{0 l} \mid \psi_{0 l}=\left(0,0, \sin \frac{l \pi(r-1)}{\left(r_{2}-1\right)}\right)^{T}, l=1,2, \ldots\right\}, \\
& \beta_{0 l} \in B_{2}=\left\{\beta_{0 l} \mid \beta_{0 l}=-\left(\frac{l \pi}{r_{2}-1}\right)^{2}, l=1,2, \ldots\right\},
\end{aligned}
$$

as $l=0, \psi_{n 0}=0$.

Now, according to the Fourier expansion, $B_{1} \cup B_{2}$ are all the eigenvalues of (4.2)-(4.7), the corresponding eigenvectors $E_{1} \cup E_{2}$ form a complete basis in $H_{1}$.

In view of the condition $\mu<-\delta$, by simple calculation, we know that $\kappa>0, \kappa r_{0}^{2}-1>0$, then

$$
\Delta \leq 0
$$

thus,

$$
\operatorname{Re} \beta_{n l}<-\varepsilon \quad(0<\varepsilon<\alpha),
$$

and

$$
\beta_{0 l}=-\left(\frac{l \pi}{r_{2}-1}\right)^{2}<0 .
$$

Now, by Lemma 4.3, the proof of Theorem 4.4 is completed.

Proof of Theorem 4.5 Utilizing the method in Theorem 4.4, we have the following results for the eigenvalue problem of $L_{\lambda}^{*}$, here $L_{\lambda}^{*}$ is adjoint operator of $L_{\lambda}$.

$$
\begin{aligned}
& \text { As }(n, l) \neq(0,0), \\
& \left\{\begin{array}{l}
C_{n l}^{*}=\frac{2 \alpha\left(\frac{1}{r_{0}^{2}}-\kappa\right) \alpha^{2} \lambda}{ \pm \sqrt{\Delta}}, \\
\beta_{n l}(\lambda)=-\alpha \pm \frac{\sqrt{\Delta}}{2 \alpha},
\end{array}\right.
\end{aligned}
$$

where

$$
\Delta=4 \alpha a^{2} \lambda^{2} \kappa\left(\frac{1}{r_{0}^{2}}-\kappa\right)
$$


the corresponding eigenvectors are as follows:

$$
\psi_{n l}^{*}=\left\{\begin{array}{l}
\frac{n l \pi^{2}}{L\left(r_{2}-1\right)} \sin \frac{n \pi z}{L} \cos \frac{l \pi(r-1)}{\left(r_{2}-1\right)} \\
\left(\frac{n \pi}{L}\right)^{2} \cos \frac{n \pi z}{L} \sin \frac{l \pi(r-1)}{\left(r_{2}-1\right)} \\
C_{n l}^{*} \cos \frac{n \pi z}{L} \sin \frac{l \pi(r-1)}{\left(r_{2}-1\right)}
\end{array}\right.
$$

satisfying

$$
\begin{aligned}
& E_{1}^{*}=\left\{\psi_{n l}^{*} \mid \psi_{n l}^{*} \text { satisfies (4.16), }(n, l) \in N \times N\right\}, \\
& B_{1}=\left\{\beta_{n l}(\lambda) \mid \beta_{n l}(\lambda) \text { satisfies (4.15), }(n, l) \in N \times N\right\} .
\end{aligned}
$$

As $n=0$,

$$
L_{\lambda}^{*} \psi_{0 l}^{*}=\beta_{0 l}(\lambda) \psi_{0 l}^{*},
$$

where

$$
\begin{aligned}
& \psi_{0 l}^{*} \in E_{2}^{*}=\left\{\psi_{0 l}^{*} \mid \psi_{0 l}^{*}=\left(0,0, \sin \frac{l \pi(r-1)}{\left(r_{2}-1\right)}\right)^{T}, l=1,2, \ldots\right\}, \\
& \beta_{0 l} \in B_{2}=\left\{\beta_{0 l} \mid \beta_{0 l}=-\left(\frac{l \pi}{r_{2}-1}\right)^{2}, l=1,2, \ldots\right\} .
\end{aligned}
$$

As $l=0, \psi_{n 0}^{*}=0$.

Consider equation $\beta_{n l}(\lambda)=0$, by (4.15), we have

$$
\begin{aligned}
& \alpha\left(\alpha+\frac{1}{r_{0}^{2}}\right)^{2}-\lambda^{2} a^{2} \kappa\left(\frac{1}{r_{0}^{2}-\kappa}\right)=0, \\
& \lambda^{2}=\frac{\alpha^{3}}{a^{2} \kappa\left(\frac{1}{r_{0}^{2}}-\kappa\right)} .
\end{aligned}
$$

Now, we have the critical Taylor number

$$
T_{c}=\min _{(n, l) \in N^{2}} \frac{\alpha^{3}}{a^{2} \kappa\left(\frac{1}{r_{0}^{2}}-\kappa\right)}
$$

through sample calculation, as $(n, l)=\left(\frac{L}{\sqrt{2}\left(r_{2}-1\right)}, 1\right), \frac{\alpha^{3}}{a^{2} \kappa\left(\frac{1}{r_{0}^{2}}-\kappa\right)}$ reaches its minimal value. We assume that $n+\frac{1}{2} \neq \frac{L}{\sqrt{2}\left(r_{2}-1\right)}$ for $\forall n>0$, then there exists only one couple $\left(n_{1}, l\right)$, making $\frac{\alpha^{3}}{a^{2} \kappa\left(\frac{1}{r_{0}^{2}}-\kappa\right)}$ reach the minimal value.

Therefore, we have

$$
\begin{aligned}
& \beta_{n_{1} l}(\lambda)= \begin{cases}<0, & \text { if } \lambda<T_{c}, \\
=0, & \text { if } \lambda=T_{c}, \\
>0, & \text { if } \lambda>T_{c},\end{cases} \\
& \beta_{n l}\left(T_{c}\right)<0, \quad \forall(n, l) \neq\left(n_{1}, 1\right) .
\end{aligned}
$$


The reduced form of (3.4) into its center manifold in $H$ is:

$$
\frac{d x}{d t}=\beta_{n_{1} 1} x+\frac{1}{\left\langle\psi_{n_{1} 1}, \psi_{n_{1}}^{*}\right\rangle_{H}}\left\langle G(\psi, \psi), \psi_{n_{1} 1}^{*}\right\rangle_{H},
$$

where $\psi \in H_{1}$ can be written by

$$
\psi=x \psi_{n_{1} 1}+\Phi
$$

$\Phi$ is a center manifold function. $G(u, v)$ is a bilinear operator, satisfying

$$
G(u, v)=-P\left((\tilde{u} \cdot \nabla) v_{z},(\tilde{u} \cdot \nabla) v_{r}-\frac{u_{\theta} v_{\theta}}{r_{0}},(\tilde{u} \cdot \nabla) v_{\theta}+\frac{u_{\theta} v_{r}}{r_{0}}\right) .
$$

Through direct calculation, we have

$$
\left\langle G\left(\psi_{n_{1} 1}, \psi_{n_{1} 1}\right), \psi_{J}^{*}\right\rangle_{H}= \begin{cases}0, & \psi_{J}^{*} \neq \psi_{2 n_{1} 2}^{*}, \\ -a_{1}^{2} b_{1} c_{11} c_{11}^{*} L\left(r_{2}-1\right), & \psi_{J}^{*}=\psi_{2 n_{1} 2}^{*},\end{cases}
$$

where,

$$
a_{1}=\frac{n_{1} \pi}{L}, \quad b_{1}=\frac{\pi}{r_{2}-1}, \quad c_{11}=C_{n_{1}}, \quad c_{11}^{*}=C_{n_{1} 1}^{*} .
$$

Here, we use the mark as follows:

$$
o(2)=o\left(x^{2}\right)+O\left(\left|\beta_{n_{1} l}(\lambda) x^{2}\right|\right), \quad o(3)=o\left(x^{3}\right)+O\left(\left|\beta_{n_{1} l}(\lambda) x^{3}\right|\right) .
$$

Then the center manifold function can be written by

$$
\Phi=-\frac{x^{2}\left\langle G\left(\psi_{n_{1} 1}, \psi_{n_{1} 1}\right), \psi_{2 n_{1} 2}^{*}\right\rangle_{H}}{\beta_{2 n_{1} 2}\left\langle\psi_{2 n_{1} 2}, \psi_{2 n_{1} 2}^{*}\right\rangle_{H}} \psi_{2 n_{1} 2}+o(2)
$$

Direct computation yields that

$$
\begin{aligned}
& \left\langle G\left(\psi_{n_{1} 1}, \psi_{2 n_{1} 2}\right), \psi_{n_{1} 1}^{*}\right\rangle_{H}=2 a_{1}^{2} b_{1} c_{11} c_{11}^{*} L\left(r_{2}-1\right), \\
& \left\langle G\left(\psi_{2 n_{1} 2}, \psi_{n_{1} 1}\right), \psi_{n_{1} 1}^{*}\right\rangle_{H}=-a_{1}^{2} b_{1} c_{11} c_{11}^{*} L\left(r_{2}-1\right), \\
& \left\langle\psi_{n_{1} 1}, \psi_{n_{1} 1}^{*}\right\rangle_{H}=\frac{1}{2} c_{11} c_{11}^{*} L\left(r_{2}-1\right), \quad\left\langle\psi_{2 n_{1} 2}, \psi_{2 n_{1} 2}^{*}\right\rangle_{H}=8 c_{11} c_{11}^{*} L\left(r_{2}-1\right) .
\end{aligned}
$$

Finally, we utilize (4.20), (4.22)-(4.24) in (4.19) to obtain

$$
\frac{d x}{d t}=\beta_{n_{1} 1} x-\sigma x^{3}+o(3)
$$

where

$$
\sigma=\frac{-1}{4 \beta_{2 n_{1} 2} a_{1}^{4} b_{1}^{2}}>0 .
$$

Now, according to Theorem 6.9 in [14], the proof of Theorem 4.5 is completed. 


\section{Competing interests}

The authors declare that they have no competing interests.

\section{Authors' contributions}

The authors typed, read and approved the final manuscript.

\section{Acknowledgements}

The authors express their sincere thanks to the referees for helpful comments and suggestions which led to the improvement of the presentation and quality of the work.

\section{Received: 26 July 2013 Accepted: 6 September 2013 Published: 07 Nov 2013}

\section{References}

1. Taylor, Gl: Experiments with rotating fluid. Proc. R. Soc. Lond. Ser. A 100, 114-121 (1921)

2. Couette, MFA: Études sur le Frottement des Liquides. Ann. Chim. Phys. 21, 433-510 (1890)

3. Mallock, A: Experiments on fluid viscosity. Philos. Trans. R. Soc. Lond. A 187, 41-56 (1896)

4. Cloes, D: Transition in circular Couette flow. J. Fluid Mech. 21, 385-425 (1965)

5. Chandrasekhar, S: Hydrodynamic and Hydromaganetic Stability. Dover, New York (1981)

6. Walowit, J, Tsao, S, DiPrima, RC: Stability of flow between arbitrarily spaced concentric cylindrical surfaces including the effect of a radial temperature gradient. J. Appl. Mech. 31, 585-593 (1964)

7. Drazin, P, Reid, W: Hydromagnetic Stability. Cambridge University Press, Cambridge (1981)

8. Velte, W: Stabilität und Verzweigung stationärer Lösungen der Navier-Stokeschen Gleichungen beim Taylorproblem. Arch. Ration. Mech. Anal. 22, 1-14 (1966)

9. Kirchgässner, K, Die Instabilität der Strömung zwischen zwei rotierenden Zylindern gegenüber Taylor-Wirbeln für beliebige Spaltbreiten. Z. Angew. Math. Phys. 12, 14-30 (1961)

10. Kirchgässner, K, Sorger, P: Branching analysis for the Taylor problem. Q. J. Mech. Appl. Math. 22, $183-209$ (1969)

11. Yudovich, VI: Secondary flows and fluid instability between rotating cylinders. J. Appl. Math. Mech. 30, 1193-1199 (1966)

12. Ma, T, Wang, S: Bifurcation Theory and Applications. World Scientific, Singapore (2005)

13. Ma, T, Wang, S: Stability and bifurcation of the Taylor problem. Arch. Ration. Mech. Anal. 181, 149-176 (2006)

14. Ma, T, Wang, S: Stability and Bifurcation of Nonlinear Evolution Equations. Science Press, Beijing (2007) (in Chinese)

15. Taylor, Gl: Stability of a viscous liquid contained between two rotating cylinders. Philos. Trans. R. Soc. Lond. A 223, 289-343 (1923)

16. Pazy, A: Semigroups of Linear Operators and Applications to Partial Differential Equations. Springer, New York (1983)

17. Adams, RA: Sobolev Spaces. Academic Press, New York (1975)

18. Wang, M: Semigroups and Evolution Equations. Science Press, Beijing (2006) (in Chinese)

\section{Submit your manuscript to a SpringerOpen ${ }^{\circ}$ journal and benefit from:}

- Convenient online submission

Rigorous peer review

- Immediate publication on acceptance

- Open access: articles freely available online

- High visibility within the field

- Retaining the copyright to your article 